\title{
Gamificación aplicada a la educación: videojuego Serpientes y Escaleras
}

\author{
José Manuel Ortiz-Ramírez \\ Egresado de Ingeniería Informática, mención Software, \\ de la Universidad a Distancia de Madrid, UDIMA \\ josemortizr@gmail.com
}

\section{Javier Bravo-Agapito}

Profesor doctor del Departamento de Ingeniería Informática de la Escuela de Ciencias Técnicas e Ingeniería de la Universidad a Distancia de Madrid, UDIMA

javier.bravo@udima.es

\section{Extracto}

La educación, en general, y las universidades, en particular, están constantemente buscando nuevas formas de trasmitir el conocimiento. En los últimos años, la tecnología ha permitido que se consiga este objetivo, haciendo que el aprendizaje sea más cercano y accesible; sin embargo, existen algunos obstáculos que la tecnología por sí sola no es capaz de superar, y ahí es donde entra en juego un concepto que, a pesar de no ser nuevo, está aún poco explotado: la gamificación.

En este trabajo se presenta un ejemplo de gamificación aplicado a la educación, el videojuego Serpientes y Escaleras, incorporando elementos psicológicos como la motivación, los estímulos o las emociones -muy habituales en los videojuegos-, que aumentan el interés y la concentración de los estudiantes en el aprendizaje de ciertas materias.

Finalmente, se realiza una retrospectiva general del proyecto y se exponen ideas para estudios futuros que puedan ampliar los objetivos de este trabajo, cuyo desarrollo se realizó en el contexto del trabajo fin de grado de Ingeniería Informática y donde se utilizó la asignatura de Autómatas y Procesadores de Lenguajes como materia de ejemplo para la enseñanza.

Palabras clave: gamificación; aprendizaje; motivación; ingeniería; videojuego.

Cómo citar: Ortiz-Ramírez, J. M. y Bravo-Agapito, J. (2019). Gamificación aplicada a la educación: videojuego Serpientes y Escaleras. Tecnología, Ciencia y Educación, 14, 149-166. 


\title{
Gamification applied to education: Snakes \& Ladders videogame
}

\author{
José Manuel Ortiz-Ramírez \\ Javier Bravo-Agapito
}

\begin{abstract}
Education, in general, and universities, in particular, are constantly searching new ways of transmitting knowledge. In last years, the technology has made it possible to achieve this goal making learning closer and more accessible, however, there are some obstacles that technology alone is not able to overcome, and that is when a concept comes in to play, despite not being new, is still insufficiently exploited: the gamification.

This article presents an example of gamification oriented to learning, the Snakes \& Ladders videogame, adding psychological elements like motivation, stimuli or emotions -very usual in videogames- that increases the student's interest and motivation in learning of some subjects. Finally, a general retrospective of the project is carried out and ideas are presented for future works that can expand the objectives of this work, whose development was carried out in the context of the final degree project in Computer Engineering and where the subject of Automata and Language Processors was used as an example for teaching.
\end{abstract}

Keywords: gamification; learning; motivation; engineering, videogame.

Citation: Ortiz-Ramírez, J. M. y Bravo-Agapito, J. (2019). Gamification applied to education: Snakes \& Ladders videogame. Tecnología, Ciencia y Educación, 14, 149-166. 


\section{Sumario}

1. Introducción

2. Aportaciones de la investigación sobre la gamificación en entornos educativos

3. Modelo teórico

3.1. Tipología de actores

3.1.1. Características de los jugadores

3.1.2. Roles del alumnado

3.1.3. Roles del profesorado

3.2. Motivación para el aprendizaje

3.2.1. Acceso a conceptos y materiales

3.2.2. Agenda de aprendizaje

3.2.3. Resolución de tareas y ejercicios

3.2.4. Incremento paulatino del grado de dificultad de las lecciones

3.2.5. Sistemas de interacción

3.3. Creación y mantenimiento de las expectativas

3.3.1. Forma de estímulo de los componentes didácticos

3.3.2. Elementos del juego

3.3.3. Sistemas de recompensa

3.3.4. Fomento de la competencia

4. Propuesta

4.1. Arquitectura del portal de gestión

4.2. Arquitectura del motor del juego

5. Resultados

6. Conclusiones

Referencias bibliográficas

Nota: este trabajo es una extensión de la ponencia presentada en el marco de la I Jornada InnovaUDIMA con Tecnología Educativa (JIUTE), organizada por la Universidad a Distancia de Madrid, UDIMA, el 11 de marzo de 2019. 


\section{Introducción}

La definición más extendida de gamificación nos la ofrecen Korn, Funk y Schmidt (2015), para quienes este concepto significa el uso de técnicas propias de los juegos fuera del entorno lúdico que mejoran la experiencia y el compromiso de los participantes.

Las causas principales del éxito de la gamificación se encuentran principalmente en aspectos como la motivación, la fidelización, las emociones, etc. Arnold (2014) establece que la gamificación permite centrar la atención y el enfoque, dos cualidades fundamentales para mejorar el rendimiento en el aprendizaje.

\section{La definición más extendida de gamificación nos la ofrecen Korn, Funk y Schmidt (2015), para quienes este concepto significa el uso de técnicas propias de los juegos fuera del entorno lúdico que mejoran la experiencia y el compromiso de los participantes}

\section{La gamificación permite centrar la atención y el enfoque, dos cualidades fundamentales para mejorar el rendimiento en el aprendizaje}

Además, las nuevas tecnologías han posibilitado en la enseñanza nuevos canales para acceder a la información y han proporcionado formas adicionales de representar modelos abstractos (Waldegg, 2002).

Uno de los elementos que se suele utilizar para aplicar gamificación es el videojuego, pero su definición es compleja. Wolf (2001) ofrece una definición por separado del término «vídeo» y otra del término «juego», que, combinadas, nos dan una idea bastante clara de su significado. Por un lado, del término «juego» se espera algún tipo de reto, alguna recompensa por superar dichos retos, un conjunto de reglas y habilidades del jugador, y, por otro lado, del término «vídeo» se entiende que el juego será monitorizado y discurrirá en un dispositivo que muestra imágenes de vídeo, como un computador, un dispositivo portátil o una máquina electrónica de juegos.

El presente trabajo pretende aprovechar las ventajas que ofrecen tanto los videojuegos como las nuevas tecnologías aplicadas a la educación para encontrar una sinergia que ayude a los estudiantes de universidad a mejorar su rendimiento académico. 


\section{Aportaciones de la investigación sobre la gamificación en entornos educativos}

Existen múltiples escenarios en los que la gamificación se ha implementado con éxito. Por ejemplo, Deterding, Sicart, Nacke, O'Hara y Dixon (2011) afirman que la gamificación puede ser utilizada en cualquier contexto, incluido los entornos productivos. Melo-Solarte y Díaz (2018) utilizan la gamificación en entornos de enseñanza a distancia y Hernández et al. (2017) lo hacen para la gestión de equipos de trabajo en Ingeniería de Software. Sin embargo, existen ejemplos de gamificación en otros ámbitos, como la medicina, la gestión de empresas, la publicidad, etc.

El presente trabajo está enfocado en el uso de la gamificación en entornos universitarios, cubriendo tanto los entornos a distancia como los presenciales. Otros trabajos relacionados con la gamificación en entornos educativos son:

- «Plataforma gamificada que permite la resolución de desafíos en el lenguaje C en el ámbito universitario» (Vera et al., 2018), cuyo objetivo es motivar a los estudiantes a realizar una tarea opcional para reforzar el aprendizaje del lenguaje de programación $\mathrm{C}^{1}$ en asignaturas de Ingeniería Informática.

- «"La profecía de los elegidos": un ejemplo de gamificación aplicado a la docencia universitaria» (Pérez-López, Rivera y Trigueros, 2017), que narra una experiencia de innovación en el aula universitaria mediante una propuesta de gamificación.

- «El potencial de la gamificación aplicado al ámbito educativo» (Díaz y Troyano, 2013), que describe los motivos por los que los videojuegos son eficaces para aplicar la gamificación.

- «Digital game-based learning for sustainable tourism education» (Tan y Okamoto, 2018), que utiliza la gamificación para mejorar el aprendizaje del turismo sostenible.

- «Gamification of employee training and development» (Armstrong y Landers, 2018), que utiliza la gamificación para el entrenamiento y la formación de empleados.

\section{Modelo teórico}

El videojuego que se ha desarrollado es el mítico Serpientes y Escaleras, en el que el jugador debe alcanzar la última celda de un tablero de 10 x 10, avanzando por el mismo con la tirada de un dado virtual y contestando preguntas relacionadas con el tema de aprendizaje. Algunas celdas contienen serpientes, que hacen retroceder al jugador, y otras, escaleras, que permiten a los participantes avanzar más rápidamente por el tablero.

${ }^{1}$ El lenguaje $C$ es un lenguaje de programación que fue creado por Kernighan y Ritchie en 1978. Es utilizado en multitud de aplicaciones informáticas y se usa en los primeros cursos de Ingeniería Informática en asignaturas como Introducción a la Programación o Metodología de la Programación. 
Para desarrollar este videojuego se ha utilizado el modelo integrado de gamificación en entornos e-learning propuesto por Torres-Toukoumidis, Romero-Rodríguez, Pérez-Rodríguez y Björk (2016), cuya taxonomía se resume en el cuadro 1. Este modelo permite estructurar y analizar las diferentes partes que forman los juegos con el fin de identificar los elementos y las motivaciones que intervienen en el proceso de enseñanza-aprendizaje.

\section{Cuadro 1. Taxonomía del modelo integrado de gamificación en entornos e-learning}

\begin{tabular}{|c|c|c|}
\hline Dimensión & Proveniencia & Indicadores \\
\hline $\begin{array}{l}\text { Tipología de } \\
\text { actores }\end{array}$ & ¿Quién? & $\begin{array}{l}\text { Características de los jugadores (target). } \\
\text { Roles del alumnado. } \\
\text { Roles del profesorado. } \\
\text { Otros actores del proceso. }\end{array}$ \\
\hline $\begin{array}{l}\text { Motivación } \\
\text { para el } \\
\text { aprendizaje }\end{array}$ & ¿Por qué? & $\begin{array}{l}\text { Acceso a conceptos y materiales. } \\
\text { Agenda de aprendizaje. } \\
\text { Resolución de tareas y ejercicios. } \\
\text { Incremento paulatino del grado de dificultad de las lecciones. } \\
\text { Sistemas de interacción. } \\
\text { Aprendizaje basado en experiencias y ejemplificaciones pragmáticas. }\end{array}$ \\
\hline $\begin{array}{l}\text { Creación y } \\
\text { mantenimiento } \\
\text { de las expecta- } \\
\text { tivas }\end{array}$ & $\begin{array}{c}\text { ¿Cómo? } \\
\text { ¿Qué? }\end{array}$ & $\begin{array}{l}\text { Forma de estímulo de los componentes didácticos. } \\
\text { Elementos de juego: niveles, retos, metas, etc. } \\
\text { Sistemas de recompensa: tabla de posiciones, medallas, puntos, etc. } \\
\text { Fomento de competencia/colaboración. }\end{array}$ \\
\hline
\end{tabular}

Fuente: Torres-Toukoumidis et al. (2016).

\subsection{Tipología de actores}

\subsubsection{Características de los jugadores}

Son estudiantes de la universidad que cursan al menos una asignatura de algún profesor adscrito a la aplicación informática propuesta en el presente trabajo. Hay que señalar que la edad del estudiante de la UDIMA se encuentra en un intervalo que va de 18 a más de 65 
años. En el caso concreto del grado en Ingeniería Informática hay una mayor proporción de varones que de mujeres. Es importante indicar que generalmente el estudiante del grado en Ingeniería Informática interacciona de forma habitual con los computadores, por lo que experimentará menos problemas de uso de la aplicación informática.

\subsubsection{Roles del alumnado}

El único rol que tienen los estudiantes es el de jugadores del videojuego.

\subsubsection{Roles del profesorado}

Los roles que se han establecido para el profesorado son:

- Definir las asignaturas, sus niveles y las preguntas/respuestas de sus asignaturas.

- Registrar a los estudiantes que pueden participar y matricularles en las asignaturas en las que pueden participar.

- Analizar los progresos de los estudiantes y el grado de conocimiento adquirido por los estudiantes.

\subsection{Motivación para el aprendizaje}

Existen varios conceptos que promueven la motivación para el aprendizaje en entornos gamificados. Algunos de los más destacados son la competitividad, el refuerzo por recompensas y, en general, el uso de un entorno lúdico para aprender a través de la diversión.

\subsubsection{Acceso a conceptos y materiales}

Todos los conceptos sobre los que el estudiante es cuestionado se deben encontrar en los manuales, en los libros, en las unidades didácticas o en cualquier otro material suministrado por la universidad a los estudiantes, independientemente de si estos participan en el sistema de gamificación o no. Por lo tanto, el sistema de gamificación no requiere de material adicional al ya existente en la propia asignatura.

\subsubsection{Agenda de aprendizaje}

El estudiante irá consolidando sus conocimientos sobre la asignatura pasando progresivamente a través de diferentes niveles de dificultad que irá alcanzando al superar 
los retos que el videojuego le propone. En todo momento el estudiante debe conocer su progreso actual y el nivel en el que se encuentra para aumentar su motivación. Dado que para alcanzar el final del juego se deben superar preguntas de todos los niveles de dificultad, es deseable que el alumno haya recibido la formación de todos los contenidos del material de enseñanza con el fin de que el juego le sirva como refuerzo para el aprendizaje y no le genere frustración.
El estudiante irá consolidando sus

conocimientos sobre la asignatura pasando progresivamente a través de diferentes niveles de dificultad que irá alcanzando al superar los retos que el videojuego le propone. En todo momento el estudiante debe conocer su progreso actual y el nivel en el que se encuentra para aumentar su motivación

\subsubsection{Resolución de tareas y ejercicios}

El videojuego se fundamenta en preguntas de tipo test con un máximo de cuatro respuestas, por lo tanto, no se pedirán tareas ni ejercicios adicionales.

\subsubsection{Incremento paulatino del grado de dificultad de las lecciones}

El videojuego está formado por tres niveles de dificultad, a saber (en orden ascendente):

- Novicio.

- Avanzado.

- Competente.

Las preguntas que el profesor introduce en cada asignatura deben especificar su grado de complejidad, eligiendo uno de estos tres niveles. Durante el juego, se utilizarán las preguntas adecuadas en función del nivel que vaya alcanzando el estudiante. Las tres primeras filas del tablero (celdas 1 a 30 ) tienen un nivel de dificultad baja, correspondientes al nivel «novicio»; las tres filas siguientes (celdas 31 a 60) son de dificultad media, correspondientes al nivel «avanzado»; y las cuatro últimas filas (celdas 61 a 100) son de dificultad alta, correspondientes al nivel «competente». Por lo tanto, a medida que el jugador avanza por el tablero, va creciendo el nivel de dificultad.

\subsubsection{Sistemas de interacción}

Para interaccionar con el videojuego se podrá utilizar el teclado, el ratón o, en los dispositivos táctiles, los dedos. 


\subsection{Creación y mantenimiento de las expectativas}

\subsubsection{Forma de estímulo de los componentes didácticos}

El funcionamiento del videojuego consiste en alcanzar la última celda de un tablero de $10 \times 10$ desplazando una ficha que indica la posición actual del jugador. Para avanzar en dicho tablero se utilizará un dado virtual y se realizarán preguntas que el estudiante debe contestar de forma acertada. El estímulo para el estudiante consiste en llegar a la última celda (100) consiguiendo la mayor puntuación posible, ya que este valor será utilizado para la clasificación del estudiante en el ranking general de la asignatura. Un ejemplo del tablero del videojuego y de la puntuación se puede ver en la figura 1.

Figura 1. Captura de pantalla del videojuego en curso

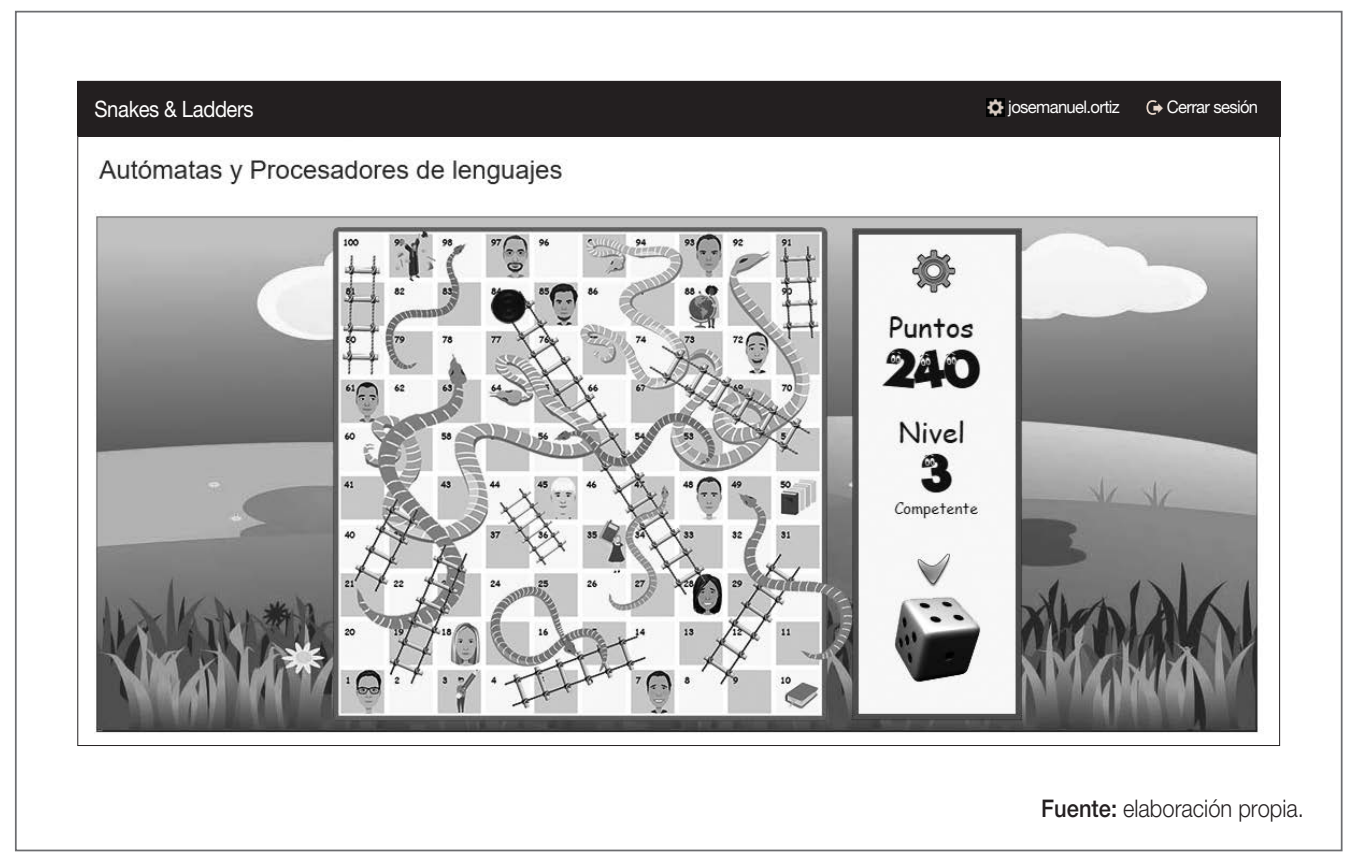

\subsubsection{Elementos del juego}

A medida que el estudiante avanza por el tablero podrá caer en celdas con escaleras o en celdas con serpientes. Las primeras permitirán avanzar hacia la celda en la que termine la escalera si el estudiante contesta correctamente a una pregunta del nivel de dificultad en el que se encuentra dicho final de la escalera. En el segundo caso, el estudiante retroce- 
derá hasta la celda donde termina la serpiente y volverá al nivel de dificultad en el que se encuentra la cola de la serpiente. La meta final se consigue cuando el estudiante llega a la última celda del tablero, momento en el que se publicarán los resultados en el ranking general. Hay que señalar que tanto el diseño del tablero como el número de escaleras y serpientes, y su ubicación en el tablero, están planteados para obtener un funcionamiento dinámico del juego y evitar que, en un número bajo de tiradas del dado, se pueda terminar el juego.

\subsubsection{Sistemas de recompensa}

A medida que el jugador va respondiendo a las preguntas, la puntuación irá reflejando el acierto o el fallo de sus respuestas. Cada respuesta acertada tendrá una recompensa de 10 puntos si está en el nivel «novicio», de 20 puntos si está en el nivel «avanzado» o de 30 puntos si está en el nivel «competente», excepto en el caso de haber caído en una escalera, en cuyo caso se aplicarán dichos puntos multiplicados por un tercio de las celdas que la escalera avance. El motivo de dividir entre 3 es porque se considera la media de saltos en cada tirada del dado que el jugador tendría que obtener en caso de avanzar sin escaleras. La siguiente ecuación representa la fórmula utilizada para dicha situación:

$$
p=\frac{\left(\text { Celda }_{\text {final }}-\text { Celda }_{\text {inicial }}\right)}{3} \times(10 \times \text { Nivel })
$$

Por ejemplo, si el jugador cae en la celda 27 (que se corresponde con el nivel 1), donde empieza una escalera que lleva hasta la celda 84 , y acierta la pregunta correspondiente, el sistema le recompensaría con:

$$
p=\frac{(84-27)}{3} \times(10 \times 1)=190 \text { puntos }
$$

Si el jugador falla la respuesta a una pregunta, el sistema descontará siempre 10 puntos independientemente de si estaba escalando una escalera o no.

\subsubsection{Fomento de la competencia}

Existirá una clasificación por asignatura con los datos de la mejor puntuación obtenida por los estudiantes que hayan jugado alguna vez y podrá ser consultada por todos los estudiantes en cualquier momento. Cada vez que se alcance la meta en una partida se actualizarán los datos del estudiante dentro del ranking general, siempre que estos sean mejores que los conseguidos en partidas anteriores. 


\section{Propuesta}

\subsection{Arquitectura del portal de gestión}

Los requisitos de la aplicación determinan el tipo de arquitectura que se debe seguir cuando se trata de diseñar el sistema informático. Obviamente, también pueden condicionar el lenguaje de programación, los patrones de diseño o las herramientas que se deben utilizar. A continuación, se enumeran algunos requisitos que se han tenido en cuenta para diseñar la arquitectura del sistema:

- Acceso a través de un navegador web. Este requisito determina que la aplicación debe ser de tipo web y que se ha de poder acceder a ella a través del protocolo HTTP.

- Persistencia de información en una base de datos. Este requisito determina que una parte de la aplicación se debe ejecutar en un servidor con acceso a un motor de base de datos.

- Mantenimiento de asignaturas, estudiantes, etc. Las páginas web que se envían al navegador deben contener información dinámica obtenida de la base de datos, por lo tanto, las páginas deberán ser formadas en la capa de servidor y enviadas a la capa de presentación.

- Videojuego. Este requisito determina la necesidad de hacer uso de un motor de juegos sobre lienzos (canvas) de HTML5 y el uso del modelo SPA (single page application) que se explicará más adelante.

Por lo tanto, de los requisitos se puede deducir la necesidad de crear una arquitectura distribuida multicapa ( $n$-tier) con una capa de presentación, una capa de lógica de negocio y una capa de acceso a datos.

La capa de presentación será la encargada de mostrar las páginas web al usuario a través de un navegador web. En esta capa se utilizarán lenguajes de marcado, como HTML, hojas de estilos CSS y lógica de cliente en lenguaje JavaScript. La capa de presentación se conectará con la capa de lógica de negocio del servidor a través del protocolo HTTP o HTTPS para solicitar los recursos necesarios.

A su vez, la capa de lógica de negocio -que se ejecutará en el servidor- será la encargada de suministrar los recursos que requiera la capa de presentación, incluyendo los ficheros de imagen o sonido, las páginas web o los datos de la base de datos que se necesiten, para lo cual, la capa de lógica de negocio podrá solicitar dicha información a la capa de acceso a datos. En el diagrama de la figura 2 se muestra un esquema general de esta arquitectura. 
Figura 2. Arquitectura multicapa

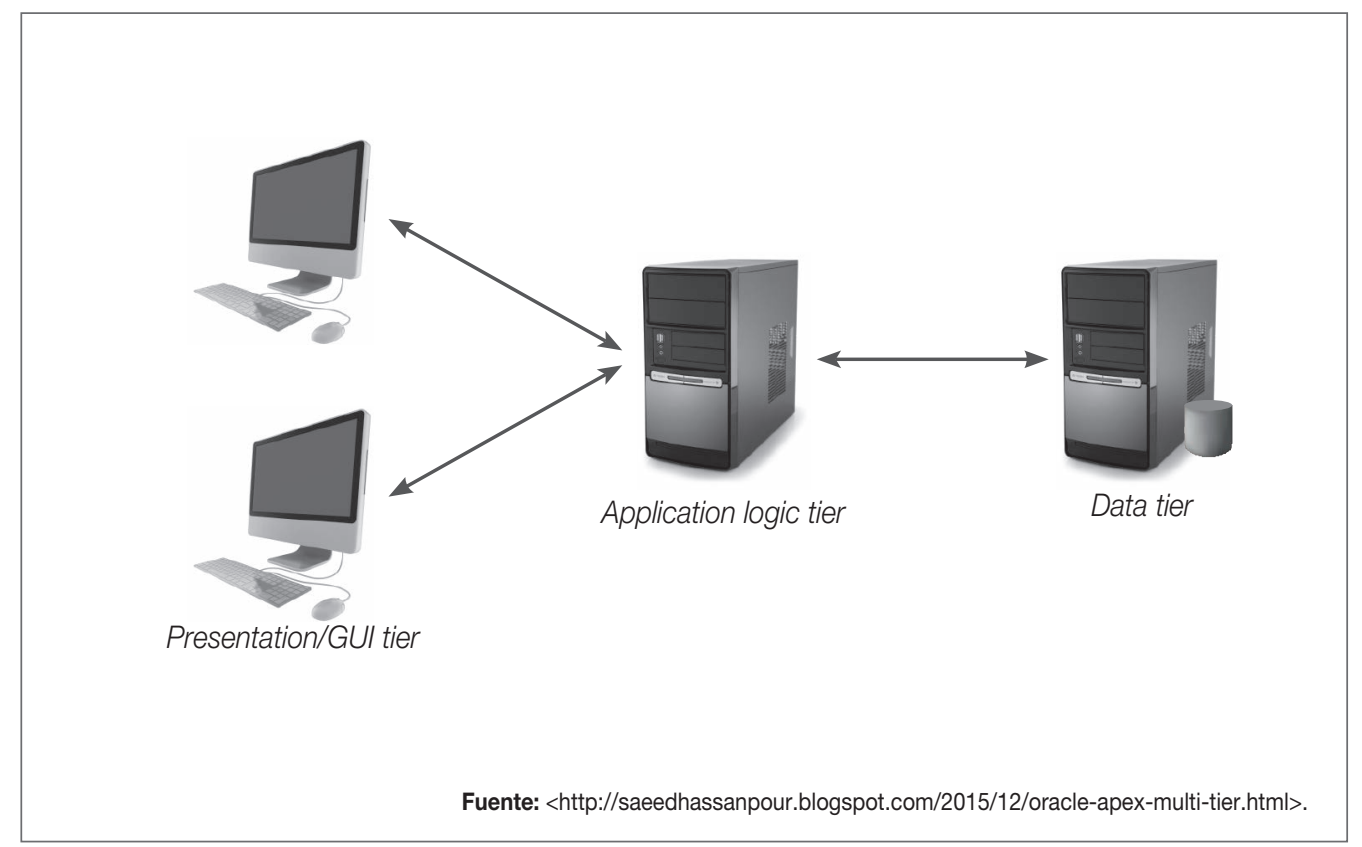

Existe un patrón de arquitectura llamado «modelo-vista-controlador» (MVC) que se adapta perfectamente a las necesidades mencionadas. MVC es un patrón de arquitectura para aplicaciones web que se caracteriza por separar la aplicación del lado del servidor en tres tipos de componentes:

- Modelo (M). Contiene los datos que se van a presentar en las páginas web. Generalmente, estos datos se obtienen de una base de datos a través de la capa de acceso a datos, se procesan a través de los controladores en la capa de lógica de negocio y se utilizan para crear las páginas web solicitadas por la capa de presentación.

- Vista (V). Define el contenido de las páginas web. En las vistas se combinan en el mismo fichero partes de código HTML, hojas de estilo y scripts de código con los modelos que nos proporciona el controlador con el fin de crear las páginas web dinámicas que nos solicitan desde el navegador. Existen muchos pseudolenguajes que se utilizan para definir las vistas debido a la necesidad de combinar el código estático de la página con los datos de los modelos. Para el proyecto de gamificación se utilizará Razor, ya que se integra de forma nativa con ASP.NET Core.

- Controlador (C). Se encarga de coordinar las solicitudes de la capa de presentación y de retornar una respuesta adecuada. Para realizar su función, el contro- 
lador procesará la lógica de negocio necesaria, accederá a la capa de acceso a datos para obtener los modelos (si es necesario) y determinará la vista que se procesará para generar la respuesta.

\section{La página web donde se desarrolla el videojuego Serpientes y Escaleras utiliza el modelo de aplicación SPA}

Por otro lado, existe una parte de la aplicación que, si bien se encuentra dentro de la arquitectura multicapa, debe tener una consideración especial. Se trata de la parte de la aplicación en la que se ejecuta el juego en sí. La página web donde se desarrolla el juego utiliza el modelo de aplicación SPA, lo cual transfiere a la capa de presentación las siguientes responsabilidades:

- Generación de la interfaz de usuario. Aunque el servidor web creará una página web inicial con todos los elementos necesarios para que se ejecute el videojuego, la capa de presentación será la encargada de crear los elementos de interfaz de usuario en cada momento del juego. Esto se conseguirá gracias al motor del juego que actuará sobre un lienzo de HTML5.

- Mantenimiento del estado. Toda la información de estado del juego (puntuación, posición de la ficha, turnos, etc.) se debe mantener en la capa de presentación.

- Acceso a datos de forma asíncrona. Cuando el juego necesite acceder al servidor para obtener/guardar información de/en la base de datos, será necesario establecer una conexión asíncrona con el mismo que no obligue al navegador a recargar una nueva página, ya que, si no, se perdería el estado actual del juego.

\subsection{Arquitectura del motor del juego}

El motor del juego es una pieza fundamental en la construcción de un videojuego. Su labor consiste en «dibujar» sobre un lienzo los diferentes elementos que intervienen en el juego en cada momento del mismo. Para realizar este proceso se utiliza la técnica del «bucle del juego", en la que un bucle de control gobierna la actualización y la representación de cada actor dentro del juego (Vallejo y Martín, 2013). Cada iteración del bucle genera una imagen que se proyecta sobre el lienzo, creando lo que se conoce como «fotograma» (frame), y una sucesión de estas imágenes creará -como si de una película se tratara- la animación del videojuego. En general, cuanto mayor sea la ratio de fotogramas por segundo (fps), mejor será la experiencia

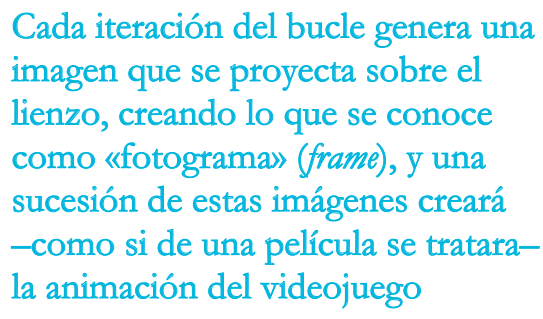

Cada iteración del bucle genera una imagen que se proyecta sobre el lienzo, creando lo que se conoce como «fotograma» (frame), y una sucesión de estas imágenes creará la animación del videojuego 
de usuario en el juego y mayor será también la exigencia computacional. Por lo tanto, el rendimiento del motor es una clave esencial del videojuego, ya que, si cada fotograma tardara demasiado en componerse, se reduciría la ratio de fotogramas por segundo y disminuiría la jugabilidad del videojuego. Esto nos obliga a elegir cuidadosamente los patrones de diseño, priorizando aquellos que estén más orientados a optimizar el rendimiento.

Las pantallas (stages) representan la etapa del juego en la que nos encontramos. Estas etapas contienen los elementos visuales necesarios para representar en la pantalla y gestionan los eventos de entrada y salida, como teclado o ratón, para que el jugador interactúe con el videojuego. Solo puede haber una pantalla activa en cada momento del juego, por lo que el motor de juego solo se tiene que preocupar de gestionar la pantalla activa.

Las pantallas están compuestas a su vez por actores. Los actores son los objetos que se dibujan finalmente en el lienzo de HTML5. Un actor puede estar compuesto a su vez por otros actores en una jerarquía de parte-todo. Una de las ventajas que tiene este modelo es que las partes de un actor tienen una posición relativa respecto de su padre, por lo que, si el actor principal se desplaza por la pantalla, las partes que lo componen se desplazarán también de forma implícita, lo cual facilita significativamente la gestión de objetos. Los actores también heredan la propiedad de visibilidad de su padre, por lo que, si ocultamos al actor principal, se ocultarán automáticamente todos sus componentes.

Otra parte importante del motor de juegos es la que gestiona los comportamientos (behaviors), ya que estos controlan la lógica final del videojuego. Su objetivo es cambiar entre las diferentes fases del juego en función de los eventos de entrada o de los estados del juego. Por ejemplo, estando en la fase de espera para tirar el dado, si se pulsa clic sobre el dado o se pulsa la tecla Espacio, el comportamiento detectará esta situa-

Otra parte importante del motor de juegos es la que gestiona los comportamientos (bebaviors), ya que estos controlan la lógica final del videojuego ción, iniciará la animación de tirar el dado, esperará al resultado, actualizará el estado con la nueva posición y cambiará a la fase de hacer una pregunta. Hay que señalar que los comportamientos del videojuego se pueden gestionar también con máquinas de estados finitos. Por ejemplo, si estamos en la fase de contestar a una pregunta, el juego no debe reaccionar si pulsamos la tecla para tirar el dado. De la misma manera, cuando estamos en la fase de tirar el dado, el juego no debe reaccionar ante la pulsación de las letras A, B, C, etc., que establecen una respuesta a un cuestionario. La máquina de estados finita nos puede ayudar a gestionar los comportamientos más adecuados en cada fase del juego con mayor facilidad.

Por último, tenemos la información del estado del videojuego, el cual, por un lado, se encarga de almacenar las variables del estado actual del juego, permitiendo conocer la puntuación, el nivel de dificultad, el número de movimientos, etc., y, por otro lado, controla las preguntas que ya se han mostrado para evitar su repetición. 


\section{Resultados}

El videojuego se aplicó en un entorno controlado y cerrado, formado por los autores de este artículo. En concreto el videojuego se aplicó en la asignatura Autómatas y Procesadores de Lenguajes del grado de Ingeniería Informática de la UDIMA. Para esta tarea se utilizó el Portal de Administración, que permite a los usuarios con el rol de profesor poder gestionar los contenidos del videojuego. En la figura 3 se puede ver la gestión de las preguntas que se realizó para la asignatura Autómatas y Procesadores de Lenguajes. Se puede observar que el portal permite editar, eliminar y asignar o modificar un nivel de cada pregunta. Además, el portal admite la importación de preguntas del sistema Moodle (véase parte superior de la figura 3), lo que posibilita la introducción de preguntas de forma fácil y eficiente. Hay que destacar que el portal también admite la posibilidad de que el profesor pueda añadir nuevas preguntas manualmente.

Figura 3. Captura de pantalla del portal de gestión

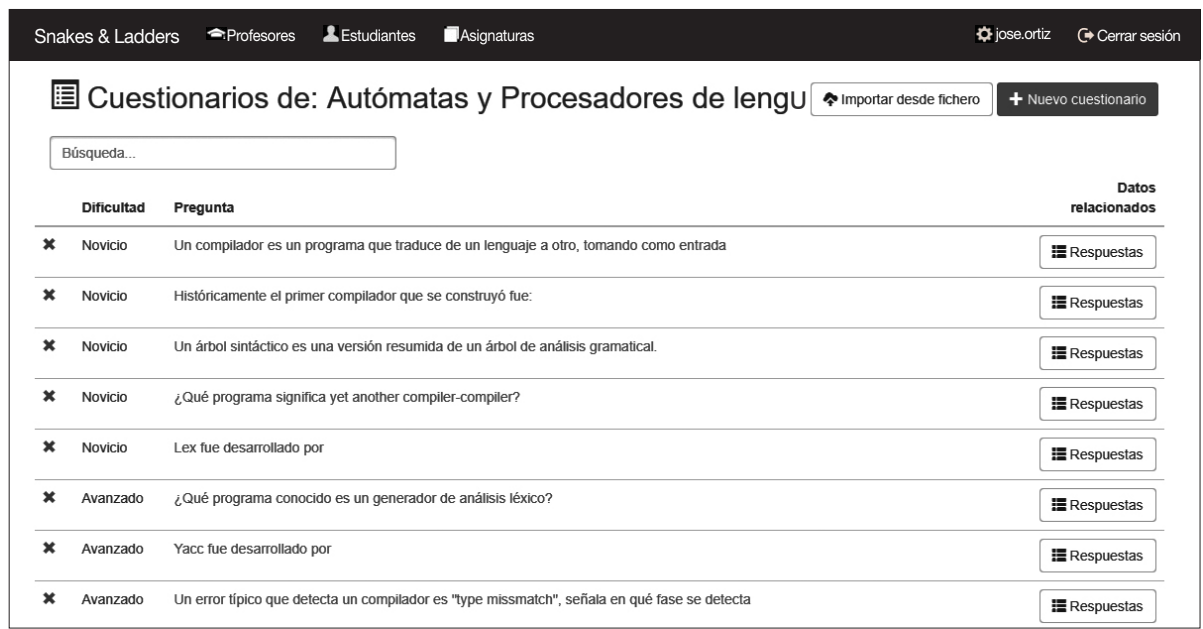

Fuente: elaboración propia.

Los autores del presente artículo crearon cuatro cuentas: dos con el rol profesor y dos con el rol estudiante. De esta forma se pudo probar cada una de las fases del videojuego con dos estudiantes, y estas pruebas permitieron mejorar la dinámica del videojuego. Volviendo a la figura 1, se puede observar una captura de pantalla de una partida en curso de uno de los estudiantes creados. En la parte central de la misma se puede observar el tablero, y en la parte lateral, las acciones del usuario. También se puede observar el diseño del tablero, que contiene 100 celdas, la disposición de las escaleras (9) y serpientes (8), y la ubicación de 
la ficha negra en la celda 84. La parte lateral derecha muestra los puntos y el nivel actuales (240 puntos y nivel 3), la imagen animada del dado virtual y una rueda que activa un menú que permite «activar/desactivar» los efectos de sonido y la música del videojuego.

En la figura 4 se puede observar el momento en el que el estudiante tiene que responder a una pregunta de la asignatura para poder avanzar en el juego. En este caso se muestra una pregunta del nivel 2 (avanzado) en la asignatura Autómatas y Procesadores de Lenguajes. Podemos ver que, una vez que el usuario responde, el videojuego le muestra con color verde si la respuesta es correcta (véase respuesta $D$ de la figura 4 en gris oscuro). En el caso de que el usuario responda de forma incorrecta, se le muestra la respuesta correcta en color verde y, en color rojo, la respuesta incorrecta que ha seleccionado. Esta codificación de colores está hecha para que el usuario (jugador) del juego, en este caso un estudiante, refuerce sus conocimientos, aunque haya fallado la pregunta.

Figura 4. Captura de pantalla de un cuestionario de la asignatura

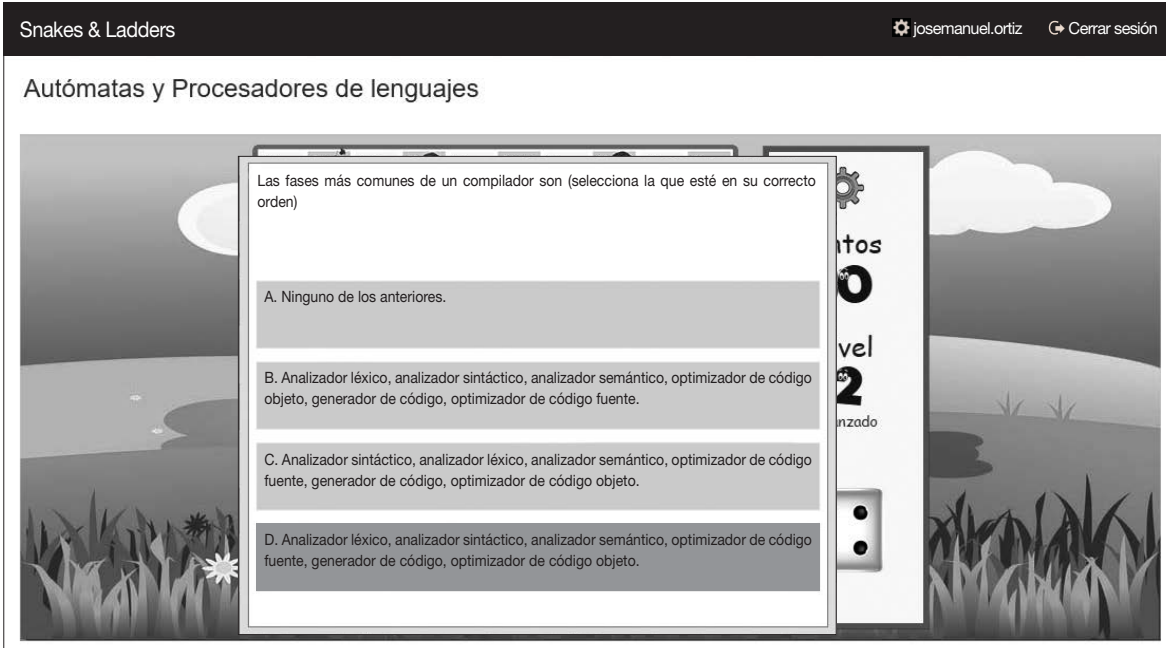

Fuente: elaboración propia.

Los resultados obtenidos de las pruebas realizadas mostraron que ambos estudiantes aumentaron su interés por la asignatura, así como también la motivación, ya que los estudiantes jugaron varias partidas seguidas con una duración media de 40 minutos con el fin de mejorar su puntuación para poder aparecer en el ranking final. No se pudo demostrar el impacto en su rendimiento académico, contenido en el aula virtual, ya que el videojuego fue diseñado como una aplicación informática externa, es decir, no dependiente del aula virtual $\mathrm{y}$, por tanto, no pudiendo ser monitorizada dentro de esta. 


\section{Conclusiones}

Este trabajo refleja una forma de aplicar la gamificación en la educación universitaria, presentando el diseño e implementación del videojuego Serpientes y Escaleras. Se han explicado las decisiones de diseño adoptadas, la tecnología utilizada y la complejidad de realizar la implementación de este videojuego. Debido a que existen muchos estudios científicos que demuestran la efectividad de la gamificación en entornos educativos, mejorando el interés y la motivación, este trabajo ha pretendido aportar un «granito» de arena al mundo de la gamificación con el fin de reducir la distancia entre los estudiantes y el conocimiento.

Hay que indicar que la gamificación del juego Serpientes y Escaleras se ha logrado transformando el juego en un videojuego, introduciendo elementos que fomentan la competitividad, como la puntuación y un ranking general, y una lógica en el juego basada en preguntas de cuestionarios. Por lo tanto, el avance o el retroceso en el juego dependen de los aciertos o de los fallos obtenidos en las preguntas. Estas van apareciendo a medida que el jugador avanza por el juego y la dificultad de las mismas varía a medida que el nivel es mayor. En este sentido, la principal ventaja consiste en dar una recompensa cuando el jugador acierta la pregunta y en mostrar la respuesta correcta cuando falla; de ese modo se consigue que el jugador aprenda al mismo tiempo que se divierte. No obstante, el videojuego no se ha podido probar en un entorno con estudiantes reales, pero sí se ha podido testar por los autores de este trabajo, obteniendo buenos resultados. De dichos resultados se puede concluir que la utilización de este videojuego puede (Arnold, 2014) aumentar la motivación y el interés por la asignatura, así como también reforzar el conocimiento de los estudiantes.

Respecto a las limitaciones cabe señalar que el videojuego funciona de forma correcta en un navegador web de un computador, pero no funciona de forma estable en dispositivos móviles como un smartphone, una tableta digital o un smartwatch. Hay que señalar también que no se ha realizado una prueba con estudiantes reales, por lo que los resultados educativos del videojuego pueden diferir de los que se obtendrían en un entorno real. Con respecto al trabajo futuro, se plantea mejorar la estabilidad en entornos móviles, implantar el videojuego en el curso académico 2019/2020 en la asignatura Autómatas y Procesadores de Lenguajes y medir el impacto en el rendimiento de los estudiantes.

\section{Referencias bibliográficas}

Armstrong, M. B. y Landers, R. N. (10 April 2018). Gamification of employee training and development. International Journal of Training and Development, 22(2), 162-169. doi: 10.1111/ijtd.12124.
Arnold, B. J. (2014). Gamification in education. American Society of Business and Behavioral Sciences Proceedings. Paris. Recuperado de <https://www.researchgate.net/profile/ Brian_Arnold3/publication/295401531_ 
Gamification_in_Education/links/56c9ea 4308ae11063709a99d.pdf> (consultado en septiembre de 2018).

Deterding, S., Sicart, M., Nacke, L., O'Hara, K. y Dixon, D. (2011). Gamification: using gamedesign elements in non-gaming contexts. Proceedings of the CHI 2011 Extended Abstracts on Human Factors in Computing Systems (pp. 2.425-2.428). Vancouver, BC, Canada: ACM.

Díaz Cruzado, J. y Troyano Rodríguez, Y. (2013). El potencial de la gamificación aplicado al ámbito educativo. III Jornadas de Innovación Docente. Innovación Educativa: Respuesta en Tiempos de Incertidumbre. Universidad de Sevilla. Recuperado de <https://idus.us. es/xmlui/handle/11441/59067> (consultado en septiembre de 2018).

Hernández, L., Muñoz, M., Mejía, J., Peña, A., Rangel, N. y Torres, C. (2017). Una revisión sistemática de la literatura enfocada en el uso de gamificación en equipos de trabajo en la ingeniería de software. Revista Ibérica de Sistemas e Tecnologias de Informação, 1(21), 33-50. doi: 10.17013/risti.21.33-50.

Korn, O., Funk, M. y Schmidt, A. (2015). Towards a gamification of industrial production: a comparative study in sheltered work environments. EICS 2015 Proceedings of the 7th ACM SIGCHI Symposium on Engineering Interactive Computing Systems (pp. 84-93). Duisburg, Alemania: ACM.

Melo-Solarte, D. S. y Díaz, P. A. (2018). El aprendizaje afectivo y la gamificación en escenarios de educación virtual. Información Tecnológica, 29(3), 237-248. doi: 10.4067/ S0718-07642018000300237.

Pérez-López, I., Rivera García, E. y Trigueros Cervantes, C. (2017). «La profecía de los elegidos»: un ejemplo de gamificación aplicado a la docencia universitaria. Revista Interna- cional de Medicina y Ciencias de la Actividad Física y el Deporte, 17(66), 243-260. doi: 10.15366/rimcafd2017.66.003.

Tan, E. y Okamoto, Y. (2018). iPlay, iLearn, iConserve: digital game-based learning for sustainable tourism education. ASEAN Tourism Research Association Conference (pp. 32-40). Phuket, Thailand: School of Arts, Murdoch University.

Torres-Toukoumidis, A., Romero-Rodríguez, L. M., Pérez-Rodríguez, M. A. y Björk, S. (2016). Modelo teórico integrado de gamificación en ambientes e-learning. Revista Complutense de Educación, 29(1), 129-145. doi: 10.5209/ RCED.52117.

Vallejo Fernández, D. y Martín Angelina, C. (2013). Desarrollo de juegos: arquitectura del motor de juegos. (2.. ed.). Ciudad Real, España: Universidad de Castilla-La Mancha.

Vera, P. M., Moreno, E. J., Rodríguez, R. A., Valles, F. E, Tamashiro, S. y Vogel, F. (2018). Plataforma gamificada que permite la resolución de desafíos en lenguaje $\mathrm{C}$ en el ámbito universitario. Libro de actas del XXWorkshop de Investigadores en Ciencias de la Computación-WICC 2018 (pp. 1.093-1.097). Corrientes, Argentina: Universidad Nacional del Nordeste. Facultad de Ciencias Exactas. Recuperado de <http://wicc2018.unne.edu. ar/wicc2018librodeactas.pdf> (consultado en septiembre de 2018).

Waldegg, G. (2002). El uso de las nuevas tecnologías para la enseñanza y el aprendizaje de las ciencias. Revista Electrónica de Investigación Educativa, 4(1). Recuperado de <https:// redie.uabc.mx/redie/article/view/53/1248> (consultado en septiembre de 2018).

Wolf, M. J. (2001). The video game as a medium. En M. J. Wolf, The medium of the video game (pp. 14-19). Austin, EE.UU.: University of Texas Press. 